OPEN ACCESS

Edited and reviewed by: Julio Navarro,

University of Victoria, Canada

*Correspondence:

Gianluca Calcagni

g.calcagni@csic.es

Specialty section:

This article was submitted to

Cosmology,

a section of the journal

Frontiers in Astronomy and Space

Sciences

Received: 29 June 2020

Accepted: 08 July 2020

Published: 02 September 2020

Citation:

Calcagni G (2020) Next Step in Gravity

and Cosmology: Fundamental Theory

or Data-Driven Models?

Front. Astron. Space Sci. 7:52.

doi: 10.3389/fspas.2020.00052

\section{Next Step in Gravity and Cosmology: Fundamental Theory or Data-Driven Models?}

\author{
Gianluca Calcagni* \\ Instituto de Estructura de la Materia, CSIC, Madrid, Spain
}

Keywords: cosmology, inflation, gravitational waves, alternative theories of gravity, modified gravity, quantum gravity

The recent detection of gravitational waves (GWs) by LIGO-Virgo (Abbott et al., 2016a) has opened a new window on the universe. Binary systems of compact objects such as black holes and neutron stars emit tiny ripples in spacetime when they inspiral and merge together. The ground-based interferometric system LIGO-Virgo has been measuring several such events and a new generation of experiments, both ground-based and space-borne, will be taking life in the next two decades, among which KAGRA (Akutsu et al., 2019) has just begun operations, LISA (Amaro-Seoane et al., 2017) is unfolding an ambitious science program, and projects such as the Einstein Telescope (Maggiore et al., 2020) and DECIGO (Seto et al., 2001; Kawamura et al., 2011, 2020) are on the table. These interferometers will also open up the possibility to detect a stochastic GW background from the early universe, let it be from inflation, from cosmic phase transitions or from alternative scenarios. This stochastic background, given by the superposition of GWs of different amplitudes and phases coming from all directions in the sky, has not been observed so far (Abbott et al., 2016b, 2018) but future missions may be able to detect it (Bartolo et al., 2016; Caprini et al., 2019; Kawamura et al., 2020; Maggiore et al., 2020).

In parallel, GWs from astrophysical sources carry a wealth of precious information about their production (via a wave-form analysis) and propagation (via a modified dispersion relation and the luminosity distance) which, in turn, depend on the underlying gravitational theory. Thus, GW astronomy is an opportunity to test general relativity as well as theories beyond it (Yunes et al., 2016; Belgacem et al., 2019).

Testing Einstein gravity is a daunting task when it comes down to calculate the wave-form of an astrophysical event made of an inspiral, a merger and a ringdown phase involving two or more compact objects. However, can we use GWs also as a tool to check other gravitational theories? The answer would be in the affirmative if interferometers had the required sensitivity to discriminate between the predictions of different theories. This depends on the specific theory or model considered: some depart from general relativity so little that any exotic effect is completely negligible as far as GWs are concerned. Others carry larger modifications and may fall within the scope of future, or even near-future, experiments.

I employed purposefully vague expressions such as "alternative scenarios" or "theories beyond general relativity" to introduce the topic of this Grand Challenge. GWs entail many challenges, each of which is grand in its own, but here we focus on a question that, as a matter of fact, transcends the realm of GWs and relates to cosmology as a whole: Should we concentrate our effort on bottom-up (i.e., data-driven) models of gravity or on top-down models stemming from fundamental theories? Before giving you my personal take on the issue, let me first clarify terminology. By bottom-up or data-driven models, I mean theoretical settings (the "up") created ad hoc to explain one or more physical phenomena (the "bottom") and that, so far, have not been embedded in any fundamental theory. An example is the class of $f(R)$ models, which have been employed both as inflationary 
and as dark-energy scenarios (De Felice and Tsujikawa, 2010; Clifton et al., 2012; Capozziello and De Laurentis, 2011). On the other hand, top-down or fundamental models are constructed in a fundamental theory of gravitation and/or particle physics (the "top") aiming to describe the elementary building blocks of geometry and matter and their interaction and, specifically, certain phenomena for which data are available (the "down"). Examples are flux-compactification models of inflation in string cosmology (reviewed in, e.g., Baumann and McAllister, 2015; Calcagni, 2017) and the bouncing cosmological scenario of loop quantum gravity (Ashtekar and Singh, 2011; Banerjee et al., 2012; Gielen and Sindoni, 2016). In both cases, a cosmological model is derived from a theory of gravitation and matter. Models only inspired by, but not fully and rigorously embedded into, fundamental theories lie between the bottom-up and the topdown extrema. Examples are the first braneworld scenarios proposed when flux compactification was not yet under control (Brax et al., 2004; Maartens and Koyama, 2010), the coeval cosmological tachyon scenarios (reviewed in Calcagni, 2017, section 13.7.2) or non-local cosmological scalars with operators resembling those found in the low-energy limit of string field theory (Aref'eva and Joukovskaya, 2005; Aref'eva, 2006). Finally, the term phenomenology can indistinctly refer either to top-down models closer than the mother theory to observed phenomena or to bottom-up models inspired by phenomena themselves.

Paradoxically, top-down models may become bottom-up. Consider the rise and fall of Hořava-Lifshitz gravity. Born as a fundamental theory breaking Lorentz invariance (Hořava, 2009), it produced a rich cosmology studied in a flood of papers. When it was noticed that classical Lorentz symmetry breaking would amplify at the quantum level to the point of ruling out the theory (Iengo et al., 2009), there were three types of reaction. A reduced group of theoreticians tried to modify the theory to avoid the problem; some lost interest in the theory; while all the rest, perhaps the majority, simply continued to do phenomenology with it, ignoring the issue.

The converse also happens and some models born as datadriven may turn out to be top-down when an embedding in a fundamental theory is discovered. An instance is Starobinsky gravity $\mathcal{L}=R+\alpha R^{2}$ (Starobinsky, 1980; Mukhanov and Chibisov, 1981). For years, it was only one among many exotic cosmological scenarios, until it came to prominence as the most favored model of primordial inflation (Ade et al., 2014; Akrami et al., 2018). Soon afterwards, it was found that Starobinsky gravity could be recovered as the local limit of a unitary and renormalizable non-local theory of quantum gravity (Briscese et al., 2014; Koshelev et al., 2016).

Starobinsky gravity is a special element of the class of $f(R)$ models. A limited number of other models may be derived from quantum gravity, for instance asymptotic safety (Bonanno, 2012; Hindmarsh and Saltas, 2012) or M-theory (Nojiri and Odintsov, 2003), but their overwhelming majority cannot be embedded in a fundamental theory. The intuitive reason is that covariant gravitational interactions include RiemannRiemann terms $R_{\mu \nu \rho \sigma} R^{\mu \nu \rho \sigma}$ and Ricci-Ricci tensor terms $R_{\mu \nu} R^{\mu \nu}$, which means that a theory made only of the Ricci scalar $R$ cannot reproduce consistently the quantum-corrected graviton propagator.

To some, this is not a problem; to me, it is. The former could argue that their foremost objective is to fit data with a bottom-up model and, once successful, one can start to think about where such model could come from. Or even not! After all, general relativity does not "come from" anywhere in particular. It does have some special properties, such as being (up to topological terms) the most general four-dimensional theory of gravitation with second-order covariant equations of motion (Lovelock, 1971, 1972). However, this is hardly an explanation of its origin. It is assumed as a fundamental description of part of Nature, and this is it. Last but not least, the advocates of the primacy of data-driven models [such as $f(R)$ gravity] could also recall that progress in physics has often been made in a bottom-up fashion, from observations to theoretical models, and from them to full-fledged theories. In a sense, people often got an interest in rigorous theories when a first crude version of them showed to predict some phenomenon not understood until then. When Newtonian celestial mechanics proved inadequate to explain the precession of Mercury, Einstein's theory of gravitation could solve the problem. Just like general relativity was, in that case, a sort of extension of Newtonian gravity, so could $f(R)$ gravity be the natural extension of general relativity when it comes to explain the late-time acceleration of the universe.

It is undeniable that $f(R)$ gravity spurred a very fecund branch of research in cosmology which gave valuable or, at times, even invaluable insights into gravity and its possible extensions. On a theoretical level, this is progress whose ramifications extended as far as string theory and other quantum gravities. However, pursuing a bottom-up model for its sake leads, in my opinion, to a dead end, even when the final outcome is positive and the model can explain data in a convincing way (i.e., per general consensus of the community). In that case, in fact, it is not guaranteed at all that it can fit into a bigger picture. Going back to my favorite scapegoat, a successful $f(R)$ would not be able to unify gravity and quantum mechanics into a theory of quantum gravity. It would not even be able to give a qualitative idea, or hints, of what such a theory should be. For example, the stability properties of quadratic gravity with only the $R^{2}$ term are very different from those of quadratic gravity with also Ricci-Ricci tensor and Riemann-Riemann terms (Kawai et al., 1998; Chiba, 2005; Núñez and Solganik, 2005). The first is not even a toy model of the latter.

Ultimately, $f(R)$ models can reproduce dark energy (Capozziello, 2002), but fail to explain it. Successful $f(R)$ models have inverse powers of the Ricci scalar and several parameters (Hu and Sawicki, 2007; Starobinsky, 2007; Capozziello and Tsujikawa, 2008; de la Cruz-Dombriz et al., 2016), not fine tuned but introduced by hand. Reversing the logic, if a bottom-up model fails to fit data, one may tailor appropriate modifications to adjust reality better, and so on, and so on. As a whole, the resulting scenario escapes the Popperian notion of science and becomes unfalsifiable.

It would be unfair to keep bullying a class of models that, after all, have been long since replaced by other, perhaps more appealing candidates. So let me go after them, too. Horndeski models and DHOST theories are now a hot topic 
among cosmologists (Langlois, 2017; Kase and Tsujikawa, 2019; Kobayashi, 2019) and they may even have a say about GWs. For instance, their effect on the GW luminosity distance of standard sirens (sources of both GWs and photons) may be large enough to reach the sensitivity threshold of LISA (Belgacem et al., 2019). Also another phenomenological model, a non-local infrared modification of gravity, can produce interestingly large deviations from general relativity (Belgacem et al., 2020). So far, none of these models has been derived from or embedded into any fundamental theory. One could wave away this criticism with a two-pronged argument: (1) since bottom-up models carry a detectable signal, they can be very useful to study the science of future GW interferometers and to stimulate research in that direction; (2) despite oft-invoked theoretical "evidence," quantum gravity is not a must and there might not be any such thing in Nature. At least, no observation so far forces this construct upon us.

We grant the latter point but, actually, the first is neither in favor nor against bottom-up models because it also applies to some quantum gravities. Reasonings loosely based on a curvature expansion of the action, or on the integration of short-scale degrees of freedom as in Wilsonian effective theory, or on continuum effective field theory ${ }^{1}$, concluding that no quantum-gravity effect can ever occur much above the Planck length scale, have been repeatedly refuted by models of inflation (reviewed in Calcagni, 2017, chapters 10, 11, 13) and GW propagation (Yunes et al., 2016; Calcagni et al., 2019) where nontrivial non-perturbative mechanisms are in action. In quantumgravity inflationary models, the accelerated expansion of the early universe boosts tiny corrections to cosmological scales leaving an imprint in the primordial scalar and tensor spectra; instances are several scenarios of string cosmology and loop quantum cosmology. Scenarios alternative to inflation also exist that fit

${ }^{1}$ See Georgi (1993) for an explanation of the difference between Wilsonian and continuum effective field theory.

\section{REFERENCES}

Abbott, B. P., Abbott, R., Abbott, T. D., Abernathy, M. R., Acernese, F., Ackley, K., et al. (2016a). Observation of gravitational waves from a binary black hole merger. Phys. Rev. Lett. 116:061102. doi: 10.1103/PhysRevLett.116.061102

Abbott, B. P., Abbott, R., Abbott, T. D., Abernathy, M. R., Acernese, F., Ackley, K., et al. (2016b). GW150914: implications for the stochastic gravitational wave background from binary black holes. Phys. Rev. Lett. 116:131102. doi: 10.1103/PhysRevLett.116.131102

Abbott, B. P., Abbott, R., Abbott, T. D., Acernese, F., Ackley, K., Adams, C., et al. (2018). GW170817: implications for the stochastic gravitational-wave background from compact binary coalescences. Phys. Rev. Lett. 120:091101. doi: 10.1103/PhysRevLett.120.091101

Ade, P. A. R., Aghanim, N., Armitage-Caplan, C., Arnaud, M., Ashdown, M., Atrio-Barandela, F., et al. (2014). Planck 2013 results. XXII. Constraints on inflation. Astron. Astrophys. 571:A22. doi: 10.1051/0004-6361/201321569

Akrami, Y., Arroja, F., Ashdown, M., Aumont, J., Baccigalupi, C., Ballardini, M., et al. (2018). Planck 2018 results. X. Constraints on inflation. arxiv: 1807.06211.

Akutsu, T., Ando, M., Arai, K., et al. (2019). KAGRA: 2.5 generation interferometric gravitational wave detector. Nat. Astron. 3, 35-40. doi: 10.1038/s41550-018-0658-y data while having some characteristic features, such as stringgas cosmology (Brandenberger, 2015) and a new version of the ekpyrotic model (Brandenberger and Wang, 2020), although they are situated more in the middle of the bottom-up/topdown continuum. In some cases, the tensor spectrum is bluetilted and it can be enhanced up to the sensitivity thresholds of GW interferometers, such as string-gas cosmology, the new ekpyrotic universe, and others (Calcagni and Kuroyanagi, 2020). Finally, although it is true that most quantum gravities predict corrections too tiny to be observed in late-time cosmological phenomena, in very few cases there is a chance (just a chance) that quantum geometry departs from the classical one enough to leave a cumulative effect on the GW luminosity distance, such as in group field theory, spin foams or loop quantum gravity (Calcagni et al., 2019).

Whether the community should focus mainly on data-driven models or on fundamental ones is a moot point: it is not an eitheror choice and many models cannot be classified as sharply as I naively tried to do here. The actual Grand Challenge, perhaps, lies in making a bigger effort in justifying the available models from the perspective of fundamental interactions, regardless of their pedigree. And, of course, to have theoreticians and phenomenologists pay more and better attention to each other.

\section{AUTHOR CONTRIBUTIONS}

The author confirms being the sole contributor of this work and has approved it for publication.

\section{ACKNOWLEDGMENTS}

The author thanks S. Capozziello, S. Nesseris, and E. Saridakis for useful comments, is supported by the I+D grant FIS201786497-C2-2-P of the Spanish Ministry of Science, Innovation and Universities, and acknowledges networking support by the COST Action CA18108.

Amaro-Seoane, P., Audley, H., Babak, S., Baker, J., Barausse, E., Bender, P., et al. (2017). Laser interferometer space antenna. arXiv [Preprint] arXiv:1702.00786.

Aref'eva, I.Ya. (2006). Nonlocal string tachyon as a model for cosmological dark energy. AIP Conf. Proc. 826:301. doi: 10.1063/1.2193132

Aref'eva, I. Ya., and Joukovskaya, L. V. (2005). Time lumps in nonlocal stringy models and cosmological applications. J. High Energy Phys. 10:087. doi: 10.1088/1126-6708/2005/10/087

Ashtekar, A., and Singh, P. (2011). Loop quantum cosmology: a status report. Class. Quant. Grav. 28:213001. doi: 10.1088/0264-9381/28/21/213001

Banerjee, K., Calcagni, G., and Martín-Benito, M. (2012). Introduction to loop quantum cosmology. SIGMA 8:73. doi: 10.3842/SIGMA.2012.016

Bartolo, N., Caprini, C., Domcke, V., Figueroa, D. G., Garcia-Bellido, J., Guzzetti, M. C., et al. (2016). Science with the space-based interferometer LISA. IV: probing inflation with gravitational waves. J. Cosmol. Astropart. Phys. 12:026 doi: 10.1088/1475-7516/2016/12/026

Baumann, D., and McAllister, L. (2015). Inflation and String Theory. Cambridge, UK: Cambridge University Press.

Belgacem, E., Calcagni, G., Crisostomi, M., Dalang, C., Dirian, Y., Ezquiaga, J. M., et al. (2019). Testing modified gravity at cosmological distances with LISA standard sirens. J. Cosmol. Astropart. Phys. 07:024. doi: $10.1088 / 1475-7516 / 2019 / 07 / 024$ 
Belgacem, E., Dirian, Y., Finke, A., Foffa, S., and Maggiore, M. (2020). Gravity in the infrared and effective nonlocal models. J. Cosmol. Astropart. Phys. 04:010. doi: 10.1088/1475-7516/2020/04/010

Bonanno, A. (2012). An effective action for asymptotically safe gravity. Phys. Rev.D 85:081503. doi: 10.1103/PhysRevD.85.081503

Brandenberger, R., and Wang, Z. (2020). Ekpyrotic cosmology with a zero-shear S-brane. arXvi2004.06437.

Brandenberger, R. H. (2015). String gas cosmology after Planck. Class. Quant. Grav. 32:234002. doi: 10.1088/0264-9381/32/23/234002

Brax, P., van de Bruck, C., and Davis, A. C. (2004). Brane world cosmology. Rep. Prog. Phys. 67:2183. doi: 10.1088/0034-4885/67/12/R02

Briscese, F., Modesto, L., and Tsujikawa, S. (2014). Super-renormalizable or finite completion of the Starobinsky theory. Phys. Rev. D 89:024029. doi: 10.1103/PhysRevD.89.024029

Calcagni, G. (2017). Classical and Quantum Cosmology. Springer.

Calcagni, G., and Kuroyanagi, S. (2020). Stochastic gravitational-wave background in quantum gravity. work in progress.

Calcagni, G., Kuroyanagi, S., Marsat, S., Sakellariadou, M., Tamanini, N., and Tasinato, G. (2019). Quantum gravity and gravitational-wave astronomy. J. Cosmol. Astropart. Phys. 10:012. doi: 10.1088/1475-7516/2019/10/012

Capozziello, S. (2002). Curvature quintessence. Int. J. Mod. Phys.D 11, 483-492. doi: 10.1142/S0218271802002025

Capozziello, S., and De Laurentis, M. (2011). Extended theories of gravity. Phys. Rep. 509, 167-321. doi: 10.1016/j.physrep.2011.09.003

Capozziello, S., and Tsujikawa, S. (2008). Solar system and equivalence principle constraints on $f(R)$ gravity by the chameleon approach. Phys. Rev.D 77:107501. doi: 10.1103/PhysRevD.77.107501

Caprini, C., Figueroa, D. G., Flauger, R., Nardini, G., Peloso, M., Pieroni, M., et al. (2019). Reconstructing the spectral shape of a stochastic gravitational wave background with LISA. J. Cosmol. Astropart. Phys. 11:017. doi: 10.1088/1475-7516/2019/11/017

Chiba, T. (2005). Generalized gravity and ghost. J. Cosmol. Astropart. Phys. 0503:008. doi: 10.1088/1475-7516/2005/03/008

Clifton, T., Ferreira, P. G., Padilla, A., and Skordis, C. (2012). Modified gravity and cosmology. Phys. Rep. 513, 1-189. doi: 10.1016/j.physrep.2012.01.001

De Felice, A., and Tsujikawa, S. (2010). $f(R)$ theories. Living Rev. Rel. 13:3. doi: 10.12942/lrr-2010-3

de la Cruz-Dombriz, Á., Dunsby, P. K. S., Kandhai, S., and Sáez-Gómez, D. (2016). Theoretical and observational constraints of viable $f(R)$ theories of gravity. Phys. Rev.D 93:084016. doi: 10.1103/PhysRevD.93.084016

Georgi, H. (1993). Effective field theory. Annu. Rev. Nucl. Part. Sci. 43, 209-252. doi: 10.1146/annurev.ns.43.120193.001233

Gielen, S., and Sindoni, L. (2016). Quantum cosmology from group field theory condensates: a review. SIGMA 12:49. doi: 10.3842/SIGMA.2016.082

Hindmarsh, M., and Saltas, I. D. (2012). $f(R)$ gravity from the renormalisation group. Phys. Rev.D 86:064029. doi: 10.1103/PhysRevD.86.064029

Hořava, P. (2009). Quantum gravity at a Lifshitz point. Phys. Rev.D 79:084008. doi: 10.1103/PhysRevD.79.084008

$\mathrm{Hu}$, W., and Sawicki, I. (2007). Models of $f(R)$ cosmic acceleration that evade solar system tests. Phys. Rev.D 76:064004. doi: 10.1103/PhysRevD.76.064004

Iengo, R., Russo, J. G., and Serone, M. (2009). Renormalization group in Lifshitztype theories. J. High Energy Phys. 11:020. doi: 10.1088/1126-6708/2009/11/020

Kase, R., and Tsujikawa, S. (2019). Dark energy in Horndeski theories after GW170817: a review. Int. J. Mod. Phys.D 28:1942005. doi: 10.1142/S0218271819420057
Kawai, S., Sakagami, M.-A., and Soda, J. (1998). Instability of 1-loop superstring cosmology. Phys. Lett.B 437, 284-290. doi: 10.1016/S0370-2693(98)00925-3

Kawamura, S., Ando, M., Seto, N., Sato, S., Musha, M., Kawano, I., et al. (2020). Current status of space gravitational wave antenna DECIGO and B-DECIGO. arXiv: 2006.13545

Kawamura, S., Ando, M., Seto, N., Sato, S., Nakamura, T., Tsubono, K., et al. (2011). The Japanese space gravitational wave antenna: DECIGO. Class. Quantum Grav. 28:094011. doi: 10.1088/0264-9381/28/9/ 094011

Kobayashi, T. (2019). Horndeski theory and beyond: a review. Rep. Prog. Phys. 82:086901. doi: 10.1088/1361-6633/ab2429

Koshelev, A. S., Modesto, L., Rachwał, L., and Starobinsky, A. A. (2016). Occurrence of exact $R^{2}$ inflation in non-local UV-complete gravity. J. High Energy Phys. 1611:067. doi: 10.1007/JHEP11(2016)067

Langlois, D. (2017). Degenerate higher-order scalar-tensor (DHOST) theories. arXiv:1707.03625.

Lovelock, D. (1971). The Einstein tensor and its generalizations. J. Math. Phys. 12:498. doi: 10.1063/1.1665613

Lovelock, D. (1972). The four-dimensionality of space and the Einstein tensor. J. Math. Phys. 13:874. doi: 10.1063/1.1666069

Maartens, R., and Koyama, K. (2010). Brane-world gravity. Living Rev. Relat. 13:5. doi: 10.12942/lrr-2010-5

Maggiore, M., van den Broeck, C., Bartolo, N., Belgacem, E., Bertacca, D., Bizouard, M. A., et al. (2020). Science case for the Einstein Telescope. J. Cosmol. Astropart. Phys. 03:050. doi: 10.1088/1475-7516/2020/ $03 / 050$

Mukhanov, V. F., and Chibisov, G. V. (1981). Quantum fluctuation and nonsingular universe. JETP Lett. 33:532.

Nojiri, S., and Odintsov, S. D. (2003). Where new gravitational physics comes from: M-theory?. Phys. Lett.B 576, 5-11. doi: 10.1016/j.physletb.2003. 09.091

Núñez, A., and Solganik, S. (2005). Ghost constraints on modified gravity. Phys. Lett.B 608, 189-193. doi: 10.1016/j.physletb.2005.01.015

Seto, N., Kawamura, S., and Nakamura, T. (2001). Possibility of direct measurement of the acceleration of the universe using $0.1-\mathrm{Hz}$ band laser interferometer gravitational wave antenna in space. Phys. Rev. Lett. 87:221103. doi: 10.1103/PhysRevLett.87.221103

Starobinsky, A. A. (1980). A new type of isotropic cosmological models without singularity. Phys. Lett. B 91, 99-102. doi: 10.1016/0370-2693(80)90670-X

Starobinsky, A. A. (2007). Disappearing cosmological constant in $f(R)$ gravity. JETP Lett. 86, 157-163. doi: 10.1134/S0021364007150027

Yunes, N., Yagi, K., and Pretorius F. (2016). Theoretical physics implications of the binary black-hole mergers GW150914 and GW151226. Phys. Rev.D 94:084002. doi: 10.1103/PhysRevD.94.084002

Conflict of Interest: The author declares that the research was conducted in the absence of any commercial or financial relationships that could be construed as a potential conflict of interest.

Copyright (c) 2020 Calcagni. This is an open-access article distributed under the terms of the Creative Commons Attribution License (CC BY). The use, distribution or reproduction in other forums is permitted, provided the original author(s) and the copyright owner(s) are credited and that the original publication in this journal is cited, in accordance with accepted academic practice. No use, distribution or reproduction is permitted which does not comply with these terms. 